\title{
The researchers investigating a new type of antimalarial
}

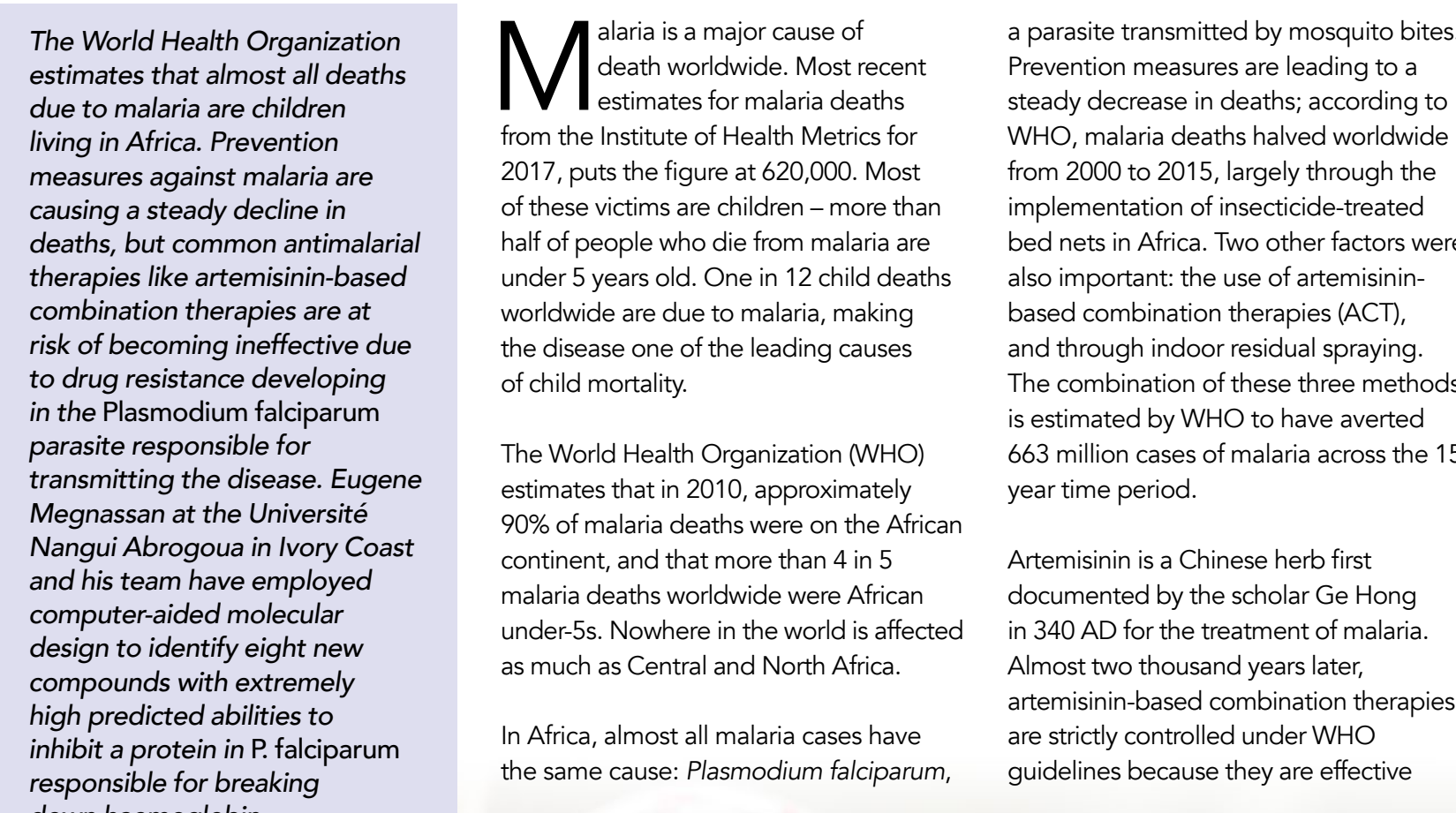

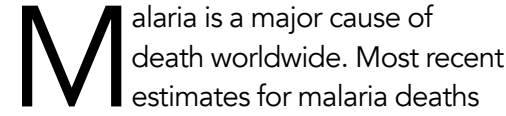
2017, puts the figure at 620,000. Most of these victims are children - more than half of people who die from malaria are under 5 years old. One in 12 child deaths worldwide are due to malaria, making the disease one of the leading causes of child mortality.

The World Health Organization (WHO) $90 \%$ of malaria deaths were on the African continent, and that more than 4 in 5 . malaria deaths worldwide were African under-5s. Nowhere in the world is affected as much as Central and North Africa.

In Africa, almost all malaria cases have the same cause: Plasmodium falciparum

a parasite transmitted by mosquito bites Prevention measures are leading to a steady decrease in deaths; according to WHO, malaria deaths halved worldwide from 2000 to 2015, largely through the implementation of insecticide-treated bed nets in Africa. Two other factors were also important. the use of artemisininbased combination therapies (ACT), and through indoor residual spraying. is combination of these three methods 663 million cases of malaria across the $15-$ year time period.

Artemisinin is a Chinese herb first documented by the scholar Ge Hong in $340 \mathrm{AD}$ for the treatment of malaria. Almost two thousand years later artemisinin-based combination therapies are strictly controlled under WHO guidelines because they are effective

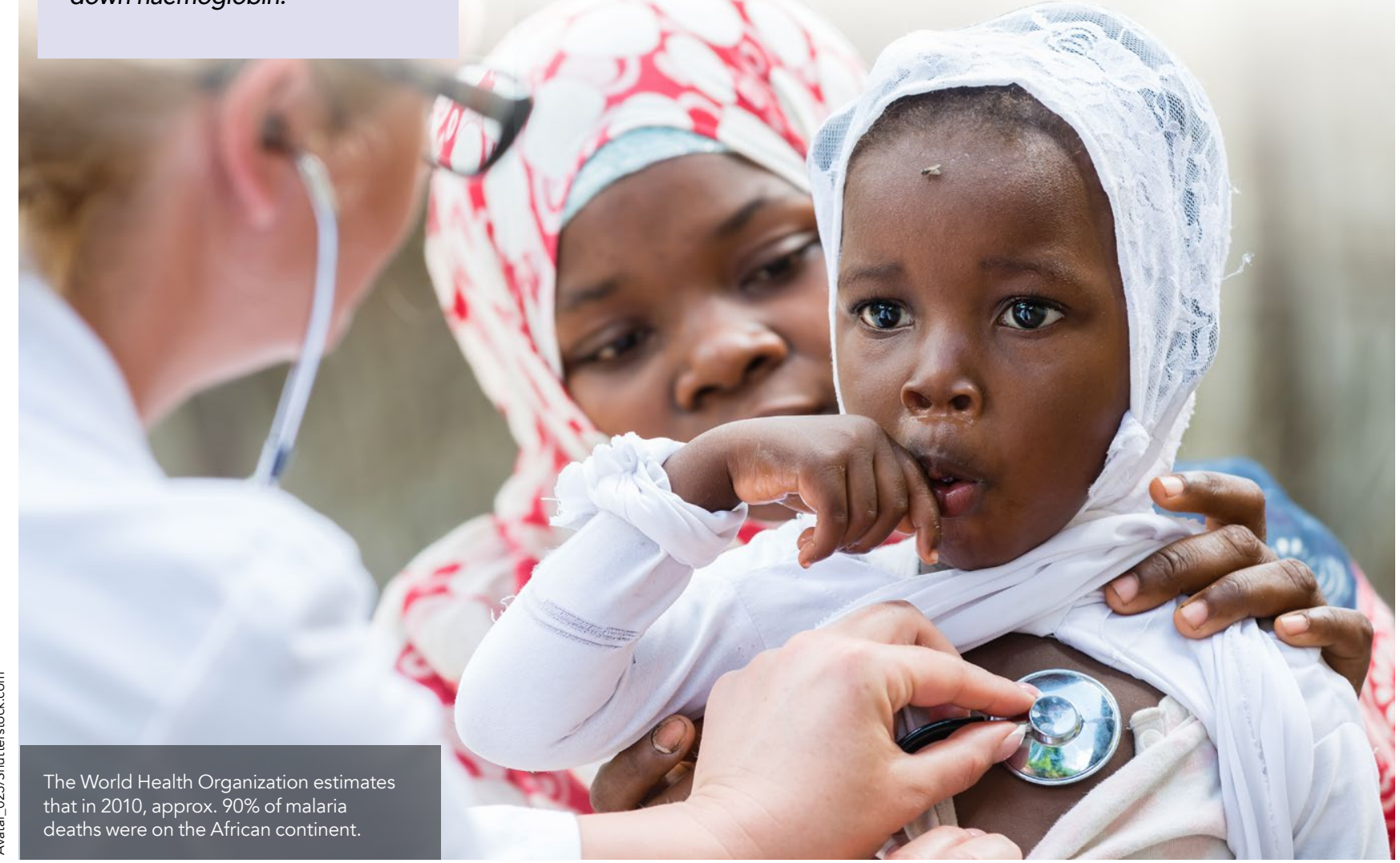

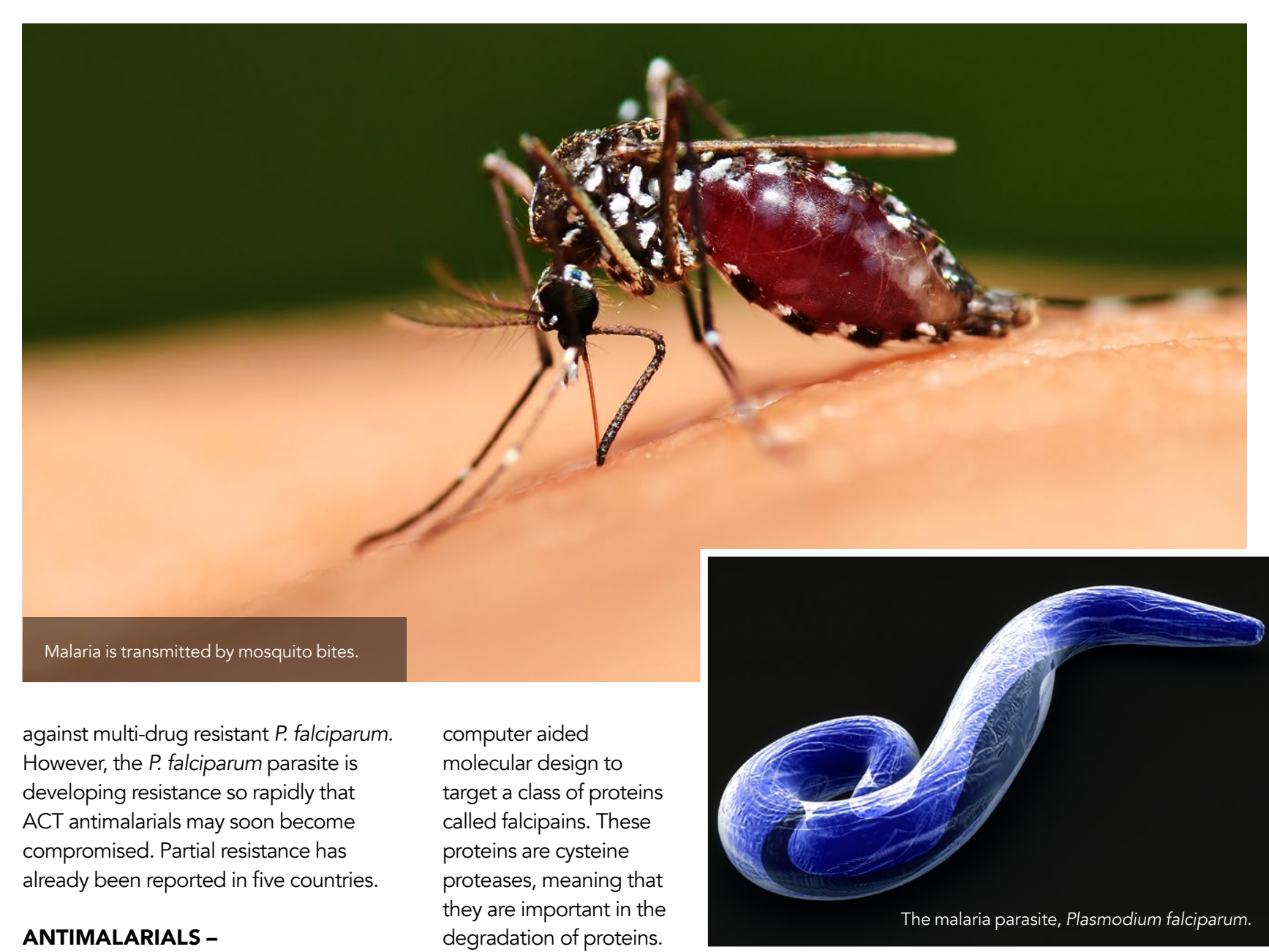

Whilst the last decade of antimalarial drug design has created some novel ACTs, many researchers are now turning to new classes of antimalarial candidates. In order to create new antimalarials, researchers need a spectic target-most of the tim specifc proteins vital to P. faliparumís cellular machinery. Knowing the structures molecular drugs. Antimalial drug design relies on knowing these structures in order to create carefully-shaped molecules which fit into the active sites of the proteins, inhibiting their ability to perfor their role in the cell.

There are relatively few known and validated structures of P. falciparum proteins which could be targets for antimalarial drugs. On top of this, there is also a lack of knowledge of the molecular motifs and features which male

Egene Megnassan at the University N Nangui Abrogoua in the lvory Coast is working with a team who are using
They are good targets

for $P$. falciparum since they are involved in the breakdown of haemoglobin whilst the paraste is invading red blood cells feature of the parasite's life-cycle

structural starting point may be a good way to begin designing a new drug. Megnassan and his team started with

The $\mathrm{P}$. falciparum parasite is developing resistance so rapidly that ACT antimalarials may soon become compromised.

and ability to invade a host, falcipains are a promising target for inhibition.

The next step in shutting down this protein is to identify a small molecule which can inhibit the falcipain protein. A type of molecule called hybrid (HLCIC) molecules target a known motif HLCICs represent good targets for the development of a new generation of However using these molecules as a each of these molecules to inhibit the falcipain-2 (FP-2) protein was available from literature sources. Each of these molecules and the FP-2 protein was modelled in $3 \mathrm{D}$ based on X-ray models were also produced of each HLCIC complexed with the FP-2 protein.

Once the structures of the proteins were accurately modelled, the models were used to predict the interactions between $\mathrm{HICIC}$ inhibitor motes. modelling identifesules. Molecular modelling identifies the most important
atoms and amino acids for the binding lactone-chalcone and isatin-chalcone in two falcipain enzymes of $P$. falciparum antimalarials, but no known HLClCs bind strongly enough to the falcipain active 


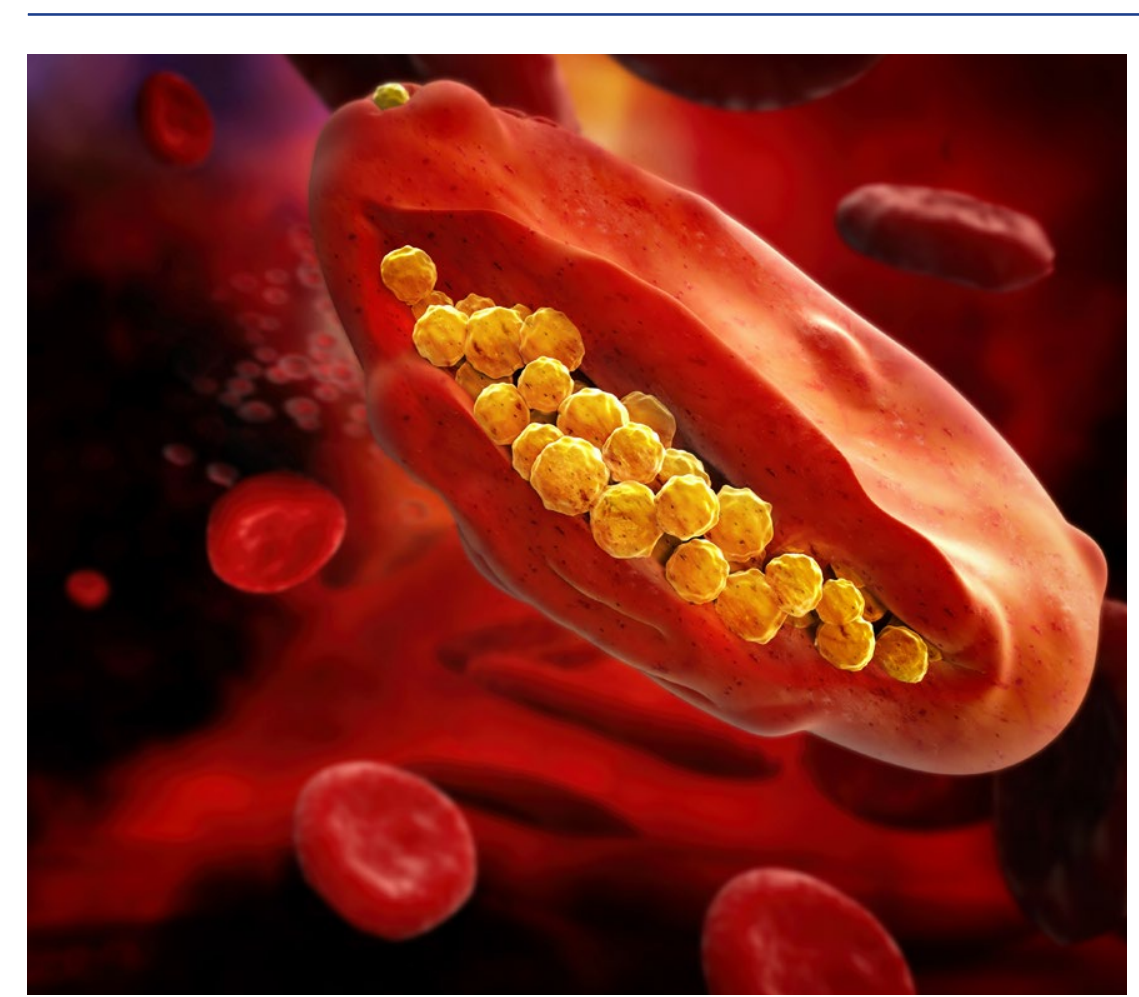

between the inhibitor and the enzyme as likely to be effective drugs, based on

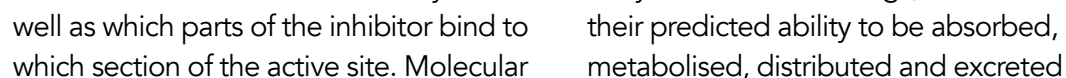
which section of the active site. Molecular simulation allowed the researchers to predict the structural changes which could with the FP-2 active site.

IDENTIFYING HLCIC ANALOGUES The researchers identified four important areas or 'pockets' in the FP-2 active site $(S 1, S 1$ ', S2 and $S 3)$, and each of these pockets needs a corresponding section when used as a drug in humans.

Twenty best-fitting inhibitor conformers were saved, and organised into 10 conformational families. The most promising molecule from each of these families was then considered for more in-depth analysis

PREDICTING INHIBITION ABILITY Each selected $\mathrm{HLCIC}$ analogue was

Megnassan and his team now have a set of promising compounds which could be taken to drug trials.

of the protein. The researchers predicted from computations that $88 \%$ of an $\mathrm{HLCIC}$ molecule's ability to inhibit the FP2 enzyme is due to how well the molecule fits these four pockets. However, by A pharmos important pocket was S2. allowed the rose search tool called PHA select compounds according to their chemical shape, giving priority to the chemical shape, giving priority to the molecules which best fill the important 52 pocket. The PH4 screening can be of new HLCIC analogues, and can give priority to compounds which are most
Predicting each molecul's Gibbs free energy of enzyme-inhibitor complex formation, which quantifies how energetically stable the HLCIC molecule can be used to predict the ability of each HLCIC analogue to inhibit FP-2.

This process led the team to the dentification of several potent HCLIC molecules, some of which are predicted o be hundreds of times more potent set of HLCIC

THE BEST CANDIDATES FOR DRUG TESTING

Megnassan and his team now have a set of promising compounds which could be taken to drug trials. Due to the importance of the S2 pocket, the top-scoring compounds are HLCIC that allows them to fill this part of the EP-2 a - 2 active site. The best of the seth about 500 times higher than the HLCICs in the training set.

Superimposing the best molecules also identifies some interesting trends. The best-designed $\mathrm{HLClC}$ analogue is on with a very bulky chain that fills $\$ 2$, a finding which matches those reported by other research groups investigatin different drugs to inhibit FP-2.

Most excitingly, the pharmacological properties of HLCICs make them good drug candidates - they should be as effectively processed by the human body as current antimalarial medication. Gastrointestnal absorption is predicted to be low, suggesting that HLCIC-type

The work done by Megnassan and his collaborators has resulted in eight novel and viable compounds for treating malaria. The team hope that the compounds will soon be synthesised and evaluated in order to check the efficacy of their design process, and guide future drug discovery of FP-2 inhibitor molecules. As currently-used antimalarials like artemisinin-based combination therapies are at risk of becoming ineffective due to drug resistance, these developments are important for the population of the Ivory The re and nearby countries in Africa. The research no hope the paper will stand as an encouragement to Africer
to invest in molecular sciences.

\section{Behind the Research}

\section{Dr Eugene Megnassan}

E: megnassaneugene.sfa@univ-na.ci E: megnase@yahoo.com
T: $+22502363008 \quad$ W: http://www. univ-na.ci

Detail

Bio

Eugene Megnassan completed graduated and received his PhD in Molecular Physics (Université de Côte d'Ivoire, U-CIV) in 1986 and Doctorate in 1998 (U-CIV) in Molecular Physics both supervised by Prof Alain Proutiere (University of Nantes), His 2 nd thesis was focused on QSPR, conformational analysis. As ICTPUNESCO associate (1999-2004) he joined ICS-UNIDO for training to CAMD (Malaria, Tuberculosis, Dengue Fever, etc.) supervised by Prof Miertus Stanislav and Vladimir Frecer and trained developing countries fellows as ICS-UNDO Consultant

\section{Research Objectives}

Eugene Megnassan's molecular physics research explores natural product-based drug discovery and early stage diagnosis at molecular and cellular level.

\section{References} Allangba, KNPG; Keita M, N'Guessan RK, Megnassan
E, Frecer V \& Miertus A. (2019) Virtual design of novel Plasmodium falciparum cysteine protease falcipain-2 hybrid lactone-chalcone and isatin-chalcone inhibitors probing the S2 active site pocket, Journal of Enzyme https://doi.org/10.1080/14756366.2018.1564288

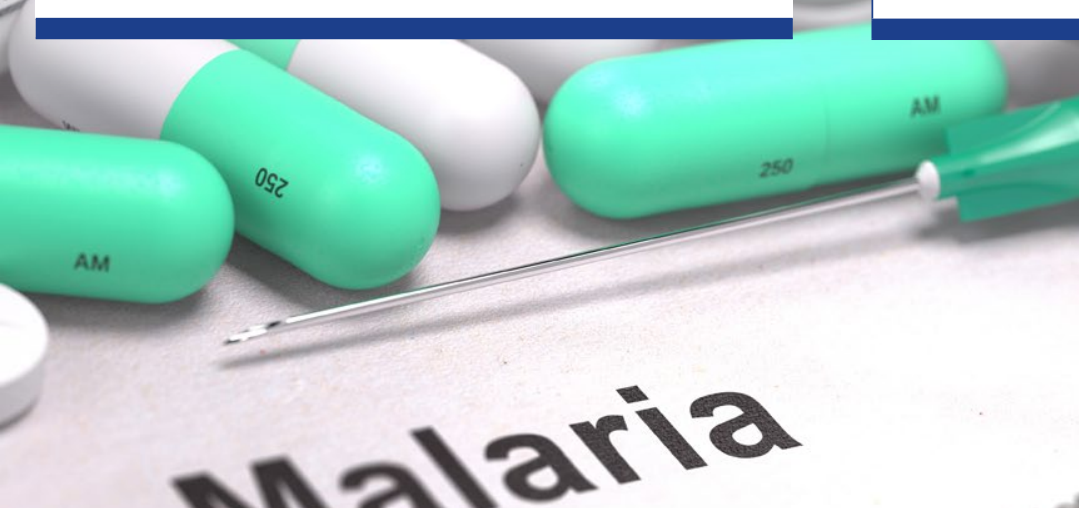
and a senior Associate, ICTP-UNESCO
Mentors

and Applied Physics Laboratory, 0294 and APVV-17-0239] Granting Agency of Slovak Ministry of Sciences [VEGA 1/0228/17]

Partners

Bioorganic Chemistry and Natural \& J. BEKRO, www.lcbosn.org) Biophysics Laboratory, University of Yaoundé ( Professor Luc
Owono Owono)

\section{Personal Response}

In this paper you provide eight HLCIC compounds which may be potential drug compounds, and also supply enzyme active site and methods for exploiting it for malaria drug design. Which of these research outco do you consider to be more impactul, and why? II Succeeding in opening the door to a better structural The nedge of WHO validated tropical disease targets. The next step is to connect the design of naturally occurring know, the best antimalarials the world has ever discovered
are natural compounds (quinine and artemisinin). II 